\title{
ANÁLISE DA DESIDRATAÇÃO DE RESÍDUOS DE PROCESSAMENTO DE MARACUJÁ-AMARELO (Passiflora edulis Flavicarpa) POR MICRO-ONDAS
}

\author{
N. C. SILVA*, L. V. D. FREITAS, T. C. SILVA, L. M. RODRIGUES, \\ C. R. DUARTE, M. A. S. BARROZO \\ Universidade Federal de Uberlândia, Faculdade de Engenharia Química \\ e-mail: neitoncsilva@yahoo.com.br
}

\begin{abstract}
RESUMO
O Brasil se destaca entre os maiores produtores mundiais de frutas, atividade essa que tem apresentado crescimento nos últimos anos. Ao mesmo tempo, tal expansão tem gerado um aumento na quantidade de resíduos, os quais muitas vezes são subaproveitados. Assim, agregar valor a estes resíduos torna-se de extremo interesse, pois é comprovado que os mesmos podem apresentar grandes quantidades de compostos importantes, como vitaminas e antioxidantes. Dentre os processos para um possível aproveitamento desse material, a desidratação tem se mostrado bastante efetiva, sendo que a utilização de micro-ondas tem se destacado devido ao seu alto desempenho, rapidez e eficiência energética quando comparada aos métodos convencionais. Sendo assim, o objetivo desse trabalho foi realizar a análise da desidratação do resíduo de maracujá por micro-ondas e avaliar os impactos dessa metodologia na remoção de umidade e nos teores de compostos fenólicos, flavonoides, ácido cítrico e ascórbico. Verificou-se que tal metodologia foi bastante eficiente na remoção de umidade, desde que realizada em potências intermediárias, como $480 \mathrm{~W}$ e também se mostrou benéfica aos compostos fenólicos, flavonoides e ácido ascórbico, mas produziu degradação do ácido cítrico. Em resumo, o uso de micro-ondas para a desidratação de resíduo de maracujá apresentou um potencial elevado para um possível aproveitamento futuro, desde que realizada sobre potências controladas, maximizando seus efeitos positivos.
\end{abstract}

\section{INTRODUÇÃO}

O Brasil se destaca mundialmente como um dos maiores produtores de frutas, sendo superado apenas por China e Índia. Esta cadeia produtiva destacou-se como um dos mais importantes segmentos econômicos do agronegócio brasileiro, com volume estimado de produção de 43,6 milhões de toneladas em 2012, onde cerca de $53 \%$ é destinada ao mercado de frutas processadas e $47 \%$ ao mercado de frutas frescas (IBRAF, 2013).

Em contrapartida a esse crescimento, há a geração de uma grande quantidade de resíduos, estimando-se que os mesmos possam representar mais de $40 \%$ do volume total de frutas processadas (SILVA, 2014). Estes resíduos, em geral, constituem uma mistura heterogênea de sementes, bagaços e cascas, cuja destinação mais comum têm sido o simples descarte ou produção de adubos.

Ao mesmo tempo, estudos recentes têm apontado que a grande maioria desse material é rica em compostos importantes para a saúde humana como ácido ascórbico, tocoferóis, carotenoides e compostos fenólicos, alguns em quantidade até mesmo superior que na polpa e sucos produzidos (WOLFE et al., 2003; MANACH et al., 2004; AJILA et al., 2007). 
Dentre as frutas processadas, destaca-se o maracujá-amarelo (Passiflora edulis Flavicarpa), que é rico em minerais e vitaminas, principalmente $\mathrm{A}$ e $\mathrm{C}$, e muito apreciado pela qualidade de seu suco, que apresenta aroma e sabor agradáveis (PEREIRA et al., 2006). O Brasil é o líder na produção mundial desse fruto, ultrapassando as 776 mil toneladas em 2012, sendo que do maracujá utilizado pelas indústrias aproveitase somente a polpa, descartando-se as cascas e sementes que podem representar de 65 a $70 \%$ do peso do fruto (OLIVEIRA et al., 2002; IBGE, 2012).

Devido à grande umidade presente no resíduo após o processamento, a desidratação, uma das mais antigas e usuais operações unitárias, destaca-se como alternativa para um possível aproveitamento desse material. A utilização de processos de desidratação em alimentos já vem sendo utilizada há muitos anos e com resultados bastante satisfatórios, já que tem se mostrado como um dos métodos mais simples, baratos e efetivos de conservação de alimentos, eliminando uma grande quantidade de água, o que inibe a proliferação de bactérias e outros microorganismos, que tornariam a vida útil (ou de prateleira) desses alimentos bastante reduzida (BORTOLOTTI, 2012).

Nos últimos vinte anos, a presença de fornos micro-ondas se tornou praticamente essencial na maioria das cozinhas. Os rápidos tempos de cozimento e maior economia de energia quando comparado aos métodos convencionais de preparo de alimentos são seus benefícios primários. Embora o seu uso para cozimento de alimentos seja bastante difundido, a aplicação dessa tecnologia para processamento de materiais é relativamente recente (VENKATESH; RAGHAVAN, 2004).

As micro-ondas têm a habilidade de penetrar os materiais e aquecê-los volumetricamente, ou seja, em toda sua extensão e de dentro para fora. Isso acontece devido à interação do campo elétrico gerado pela radiação com as moléculas de água presentes no material, através de mecanismos dipolares e iônicos (DATTA; ANANTHESWARAN, 2001).

Dessa forma, é possível obter uma desidratação rápida, mais uniforme e energeticamente eficiente quando comparada aos métodos convencionais. Além disso, o uso de micro-ondas pode impactar em sabor e aspectos nutricionais de alimentos em menor escala quando comparado aos métodos convencionais (VADIVAMBAL; JAYAS, 2010). E devido ao fato da energia ser concentrada em um único sistema, o microondas ocupa apenas 20-35\% do espaço requerido por um secador convencional (MASKAN, 2000).

$\mathrm{O}$ uso de micro-ondas em indústrias alimentícias inclui descongelamento, desidratação, cozimento, branqueamento, pasteurização, esterilização, têmpera, dentre outros (METAXAS; MERDEDITH, 1983; DATTA; ANANTHESWARAN, 2001; GUPTA; WONG, 2007). Dentre os trabalhos com frutas pode-se citar sua aplicação na desidratação de banana (MASKAM, 2000; MOUSA; FARID, 2002), pêra (ARBALLO et al., 2010), frutas cítricas (GHANEN, 2012) e maçã (ZAREIN et al, 2015).

Dessa forma, utilizar essa metodologia e avaliar os impactos de suas variáveis na eficiência do processo de desidratação e nas quantidades de compostos presentes no resíduo de maracujá pode indicar um potencial aproveitamento do mesmo e por conseguinte uma melhor aplicação desse material, permitindo economia de energia e matéria-prima, além de permitir um uso mais nobre desse material na produção de alimentos.

\section{MATERIAL E MÉTODOS}

\subsection{Resíduo utilizado}


O material utilizado nos testes foi resíduo do processamento de maracujáamarelo, fornecido pela empresa Lotus Soluções Ambientais, localizada no munícipio de Araguari-MG. Tal resíduo é bastante heterogêneo, constituído por cascas, sementes, albedo e restos de polpa.

\subsection{Aparato Experimental}

A desidratação do maracujá foi realizada em um sistema de micro-ondas acoplado com balança, conforme mostrado na Figura 1. Esse sistema consiste em um suporte de aço inox sob o qual foi colocado um micro-ondas doméstico da fabricante Panasonic, modelo NN-SF560WRU e potência nominal de $800 \mathrm{~W}$. Na parte superior foi colocada uma balança da fabricante Shimadzu, modelo AUX220 e com precisão de $10^{-4} \mathrm{~g}$, a qual permitiu o monitoramento da desidratação do resíduo simultaneamente aos experimentos.

As amostras eram colocadas sobre um suporte suspenso, conectado à parte inferior da balança através de um orifício presente na parte superior do micro-ondas. Utilizou-se $120 \mathrm{~g}$ de amostra em cada experimento e a leitura das pesagens era realizada a cada 30 segundos.

Figura 1 - Sistema de micro-ondas utilizado
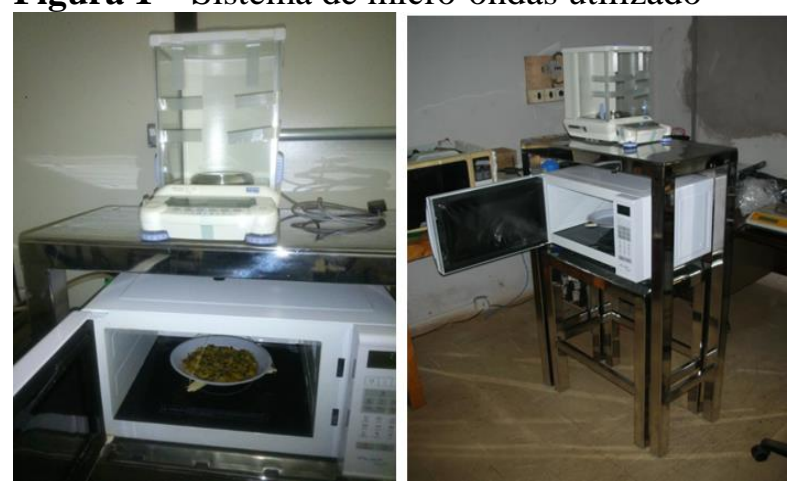

Os experimentos foram organizados em um planejamento fatorial em quatro níveis, sendo as variáveis potência (Watts) e tempo (minutos), ambas reguladas no display do equipamento, o que totalizou 16 experimentos, como pode ser observado na Tabela 1.

Tabela 1 - Planejamento Experimental.

\begin{tabular}{ccc}
\hline Experimentos & $\begin{array}{c}\text { Potência } \\
\text { (Watts) }\end{array}$ & $\begin{array}{c}\text { Tempo } \\
\text { (minutos) }\end{array}$ \\
\hline $\mathbf{1}$ & 280 & 5 \\
$\mathbf{2}$ & 280 & 10 \\
$\mathbf{3}$ & 280 & 15 \\
$\mathbf{4}$ & 280 & 20 \\
$\mathbf{5}$ & 480 & 5 \\
$\mathbf{6}$ & 480 & 10 \\
$\mathbf{7}$ & 480 & 15 \\
$\mathbf{8}$ & 480 & 20 \\
$\mathbf{9}$ & 600 & 5 \\
$\mathbf{1 0}$ & 600 & 10 \\
$\mathbf{1 1}$ & 600 & 15 \\
$\mathbf{1 2}$ & 600 & 20 \\
$\mathbf{1 3}$ & 800 & 5 \\
$\mathbf{1 4}$ & 800 & 10 \\
$\mathbf{1 5}$ & 800 & 15 \\
$\mathbf{1 6}$ & 800 & 20 \\
\hline
\end{tabular}

\subsection{Análise de Compostos Bioativos}

Tanto o resíduo in natura (antes da desidratação) quanto o resíduo desidratado passaram por uma série de análises físicoquímicas para avaliar a presença de alguns compostos bioativos nos mesmos. Realizou-se as seguintes análises:

$\checkmark$ Teor de Fenólicos Totais (TPC):

Determinado por leitura em espectrofotômetro através do método de Folin-Ciocalteau descrito por Singleton; Rossi (1965) e expresso em mg de ácido gálico/100 g de amostra em base seca;

$\checkmark$ Teor de Flavonoides Totais (TFC): Determinado pelo método colorimétrico descrito por Zhishen et al. (1999) e leitura em espectrofotômetro. Foi expresso em $\mathrm{mg}$ de rutina/100 $\mathrm{g}$ de amostra em base seca;

$\checkmark$ Teor de Acidez (TA): Obtido através de titulação com $\mathrm{NaOH}$ e expresso em $\mathrm{mg}$ de ácido cítrico/100 g de amostra em base seca (AOAC, 1995);

$\checkmark$ Teor de Ácido Ascórbico (AA): Obtido por titulometria, através da redução do 
2,6-diclorofenol-indofenol, com os resultados expressos em $\mathrm{mg}$ de ácido ascórbico/100 g de amostra em base seca (AOAC, 1995).

A umidade final das amostras foi calculada através do método de estufa, onde o material foi submetido à $105{ }^{\circ} \mathrm{C} \pm 3{ }^{\circ} \mathrm{C}$ por 24 horas.

\subsection{Microscopia Eletrônica de Varredura (MEV)}

Visando uma melhor análise do resíduo após a desidratação, as amostras também foram submetidas à microscopia eletrônica de varredura (MEV). As análises foram realizadas em microscópio eletrônico da fabricante Carl Zeiss, modelo EVO MA 10, após as amostras terem sido fixadas em stubs com fita de carbono e recobertas com ouro no metalizador da marca Leica, modelo SCD 050. A voltagem de aceleração utilizada foi de $10 \mathrm{KV}$, proporcionando ampliações de 500 vezes do material analisado.

\section{RESULTADOS E DISCUSSÃO}

\subsection{Cinética de Remoção de Umidade}

Os resultados obtidos de umidade final para cada experimento estão expressos na Tabela 2. Através dos mesmos foi possível verificar o impacto de cada potência na retirada de umidade, sendo que conforme os valores de potência foram sendo elevados foi possível obter uma maior retirada de umidade. Nas potências de 480, 600 e $800 \mathrm{~W}$, dentro do limite de 20 minutos, foi possível obter um produto final com teores de umidade abaixo de $10 \%$, considerada ideal para armazenamento do material (REY, 1964).

As curvas de MR em função do tempo estão expressas na Figura 2 e comprovaram o que foi discutido anteriormente.
Tabela 3 - Umidade final para cada experimento

\begin{tabular}{ccccc}
\hline \multirow{2}{*}{ POT } & \multicolumn{4}{c}{ TEMPO (minutos) } \\
\cline { 2 - 5 } & $\mathbf{5}$ & $\mathbf{1 0}$ & $\mathbf{1 5}$ & $\mathbf{2 0}$ \\
\hline \multirow{2}{*}{$\mathbf{2 8 0} \mathbf{W}$} & $\begin{array}{c}78,75 \% \\
( \pm 0,95)\end{array}$ & $\begin{array}{c}76,19 \% \\
( \pm 1,45)\end{array}$ & $\begin{array}{c}71,23 \% \\
( \pm 0,71)\end{array}$ & $\begin{array}{c}63,87 \% \\
( \pm 0,72)\end{array}$ \\
\hline \multirow{2}{*}{$\mathbf{4 8 0} \mathbf{W}$} & $\begin{array}{c}75,54 \% \\
( \pm 2,51)\end{array}$ & $\begin{array}{l}62,03 \% \\
( \pm 1,89)\end{array}$ & $\begin{array}{c}41,78 \% \\
( \pm 1,31)\end{array}$ & $\begin{array}{c}8,95 \% \\
( \pm 0,84)\end{array}$ \\
\hline \multirow{2}{*}{$\mathbf{6 0 0} \mathbf{W}$} & $\begin{array}{c}71,08 \% \\
( \pm 0,83)\end{array}$ & $\begin{array}{l}48,43 \% \\
( \pm 1,96)\end{array}$ & $\begin{array}{c}16,62 \% \\
( \pm 0,53)\end{array}$ & $\begin{array}{c}4,36 \% \\
( \pm 0,08)\end{array}$ \\
\hline \multirow{2}{*}{$\mathbf{8 0 0} \mathbf{W}$} & $66,60 \%$ & $25,48 \%$ & $8,23 \%$ & $5,26 \%$ \\
& $( \pm 1,41)$ & $( \pm 0,93)$ & $( \pm 0,30)$ & $( \pm 0,16)$ \\
\hline
\end{tabular}

Figura 2 - Cinética de remoção de umidade

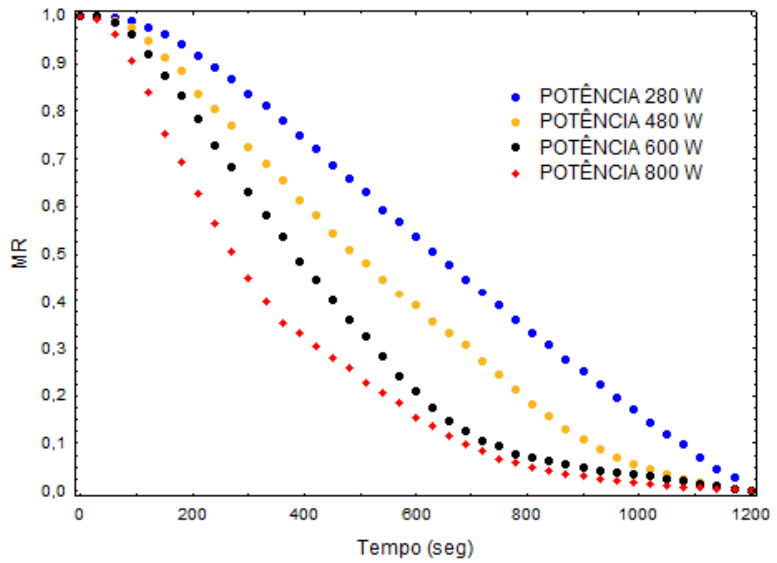

Li et. al (2010) obtiveram comportamento semelhantes para a desidratação de maçãs em micro-ondas e Zarein et al. (2015) confirmaram a eficiência dessa metodologia ao verificaram o aumento na difusividade da água através de amostras de maçã conforme a potência de micro-ondas foi sendo aumentada.

\subsection{Compostos Bioativos}

A umidade obtida para o resíduo in natura foi de $82,88 \pm 2,30 \%$, considerada bastante elevada e o que reforçou ainda mais a necessidade do uso de técnicas de desidratação para um posterior aproveitamento satisfatório desse material.

Os resultados encontrados para os compostos bioativos nessa condição estão expressos na Tabela 3. Tais resultados foram utilizados como base de comparação para os experimentos realizados, permitindo a análise dos impactos do uso das micro-ondas nos 
mesmos, sob as diferentes condições verificadas.

Tabela 3 - Compostos bioativos do resíduo de maracujá in natura

\begin{tabular}{cc}
\hline Análise & $\begin{array}{c}\text { Resultados (mg composto/ } \\
\mathbf{1 0 0} \text { g amostra seca) }\end{array}$ \\
\hline Teor de Fenólicos & $119,87 \pm 4,71$ \\
Totais (TPC) & mg ácido gálico $/ 100 \mathrm{~g}$ \\
\hline Teor de & $0,47 \pm 0,05$ \\
Flavonoides (TFC) & mg rutina $/ 100 \mathrm{~g}$ \\
\hline Teor de Acidez & $2701,35 \pm 89,13$ \\
(TA) & mg ácido cítrico $/ 100 \mathrm{~g}$ \\
\hline Teor de Ácido & $0,54 \pm 0,05$ \\
Ascórbico (AA) & mg ácido ascórbico $/ 100 \mathrm{~g}$ \\
\hline
\end{tabular}

\subsubsection{Teor de Compostos Fenólicos (TPC)}

A Figura 3 apresenta os resultados obtidos para os teores de compostos fenólicos obtidos nas desidratações realizadas. De acordo com os valores encontrados, observouse que a exposição à radiação de micro-ondas gerou resultados positivos para esses compostos, pois obteve-se para todas as potências valores de TPC próximos ou superiores aos do resíduo in natura.

Tal aumento pode ser justificado pelo rompimento da matriz interna do resíduo, conforme a umidade foi sendo removida do mesmo, o que acabou levando a liberação desses compostos no material desidratado (CHANG et al., 2006).

Figura 3 - TPC para as desidratações por microondas: $280 \mathrm{~W}$ (a), $480 \mathrm{~W}$ (b), $600 \mathrm{~W}$ (c) e $800 \mathrm{~W}(\mathrm{~d})$.

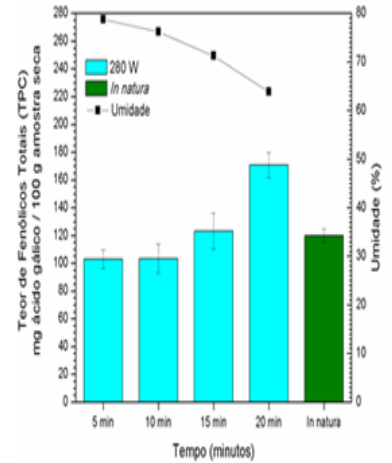

(a)

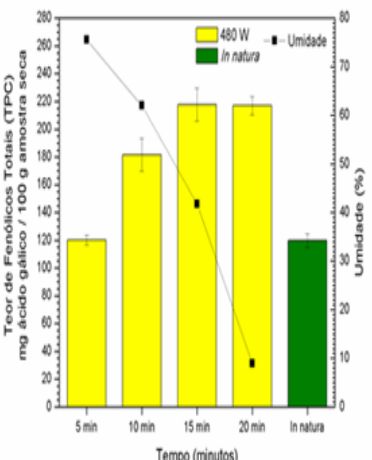

(b)

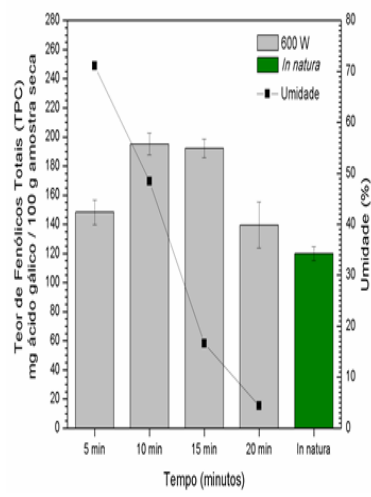

(c)

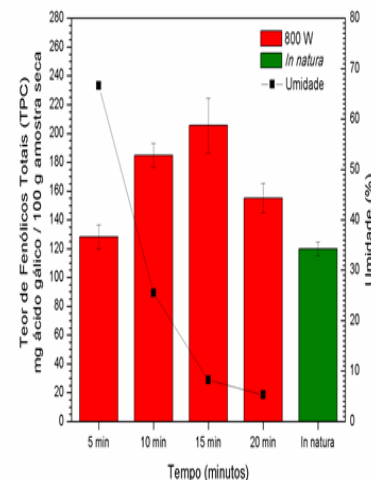

(d)
Verificou-se ainda que para as potências de 280 e $480 \mathrm{~W}$ (a e b), onde o aquecimento e, por conseguinte, a retirada de umidade, foram mais lentos, o TPC iniciou a desidratação com valores próximos aos do in natura, mas conforme o aquecimento se seguiu, tais valores se elevaram e ficaram praticamente constantes.

Já nos casos em que o fornecimento de energia foi mais intenso, como nas potências de $600 \mathrm{~W}$ e $800 \mathrm{~W}$, o TPC apresentou um aumento e atingiu um pico de concentração, mas com a continuação no fornecimento de energia e logo, excesso de aquecimento, acabou por ocorrer a degradação desses compostos, reduzindo seus teores.

Dessa forma, os melhores resultados para compostos fenólicos foram obtidos na potência de $480 \mathrm{~W}$, onde foi possível obter os teores mais elevados e ao mesmo tempo uma boa retirada de umidade.

\subsubsection{Teor de Compostos Flavonoides (TFC)}

Como pode ser observado na Figura 4, a desidratação por micro-ondas também atuou de forma positiva nos compostos flavonoides, obtendo-se valores consideravelmente superiores aos do resíduo in natura para as potências onde houve maior remoção de umidade. A justificativa para tal comportamento, mais uma vez, encontra-se no rompimento da estrutura interna do material que levou a liberação desses 
compostos durante a secagem, como observado por Chang et al. (2006).

Todavia, o comportamento desses compostos foi levemente diferente dos obtidos para os fenólicos, pois o TFC não se mostrou tão sensível ao excesso de exposição às micro-ondas, apresentando valores sempre crescentes conforme a energia é fornecida e no caso de excesso da mesma, se estabilizaram no valor máximo.

Figura 4 - TFC para as desidratações por microondas: $280 \mathrm{~W}$ (a), $480 \mathrm{~W}$ (b), $600 \mathrm{~W}$ (c) e $800 \mathrm{~W}(\mathrm{~d})$.

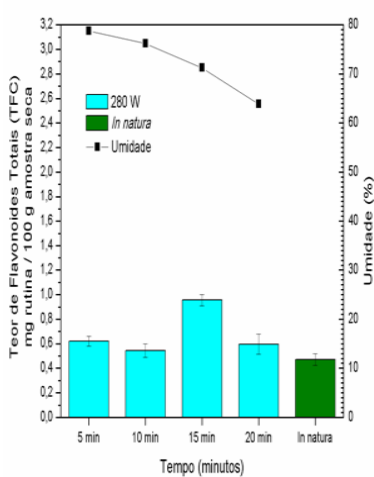

(a)

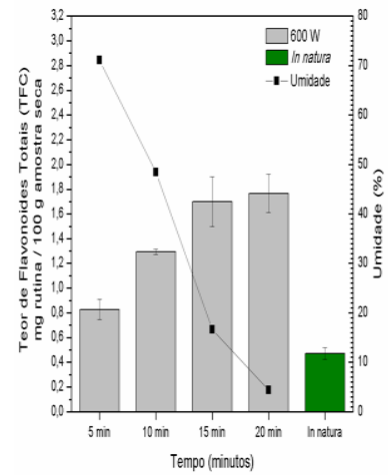

(c)

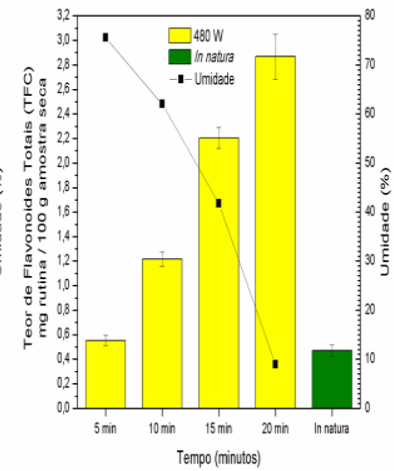

(b)

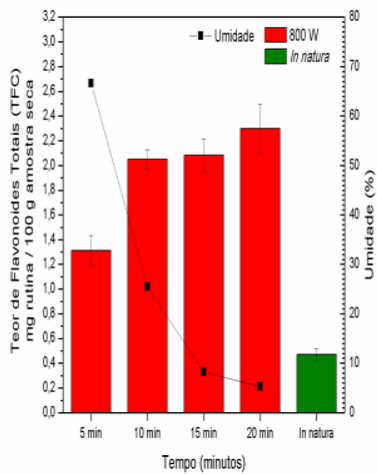

(d)
Comparando cada potência, observou-se que os melhores resultados foram obtidos também para a potência de $480 \mathrm{~W}$ (b), onde atingiu-se os valores máximos de TFC. Apesar das potências de $600 \mathrm{~W}$ (c) e $800 \mathrm{~W}$ (d) também terem apresentado bons resultados, seus resultados foram inferiores aos da potência de $480 \mathrm{~W}$, indicando que o excesso de energia decorrente desses aquecimentos levou a uma degradação simultânea a retirada dos flavonoides da estrutura do material, resultando em um valor final de TFC inferior, apesar de ainda muito elevado quando comparado ao in natura.

\subsubsection{Teor de Acidez (TA)}

Os teores de ácido cítrico obtidos para cada experimento estão apresentados na Figura 5.

Figura 5 - TA para as desidratações por microondas: $280 \mathrm{~W}$ (a), $480 \mathrm{~W}$ (b), $600 \mathrm{~W}$ (c) e $800 \mathrm{~W}(\mathrm{~d})$.

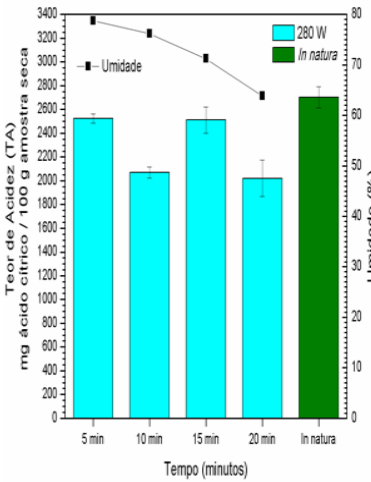

(a)

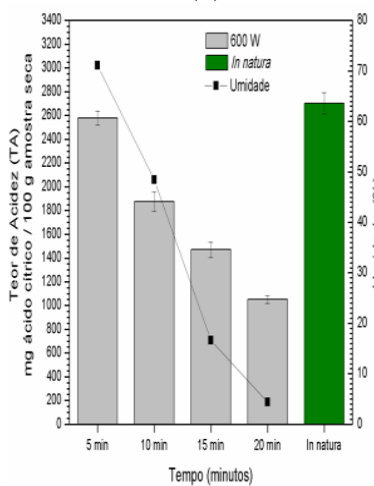

(c)

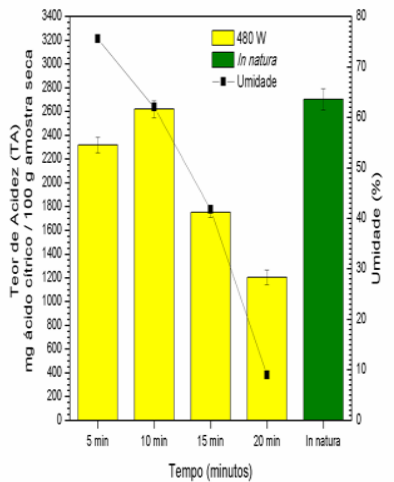

(b)

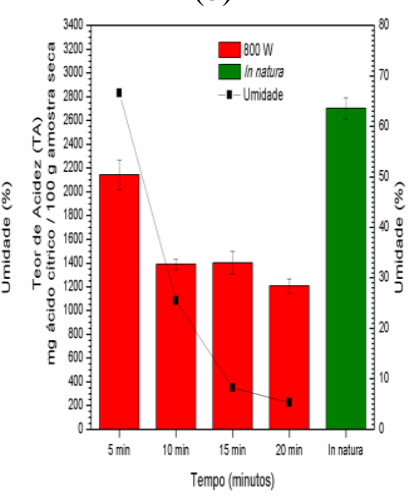

(d)
Diferentemente do ocorrido com os compostos fenólicos e flavonoides, observouse que houve impacto negativo da exposição à radiação de micro-ondas, gerando a degradação desse composto em relação ao resíduo in natura. Tal resultado foi esperado, pois conforme observado por Podsedek (2007), o ácido cítrico apresenta alta 
sensibilidade em operação a temperaturas elevadas.

Avaliando-se cada potência, observouse que para a potência de $280 \mathrm{~W}$ (a), os valores de TA oscilaram próximo ao valor do resíduo in natura, mas após isso, conforme as potências e tempos foram aumentando, os teores se reduziram drasticamente.

Dessa forma, em termos de qualidade do material e retirada de umidade, o melhor valor obtido foi novamente para a potência de $480 \mathrm{~W}$, onde foi possível obter um TA que apesar de ser o menos impactado de todas as potências, ainda representou apenas cerca de $45 \%$ do teor de ácido cítrico original do resíduo, com uma maior redução de umidade em relação a menor potência.

\subsubsection{Teor de Ácido Ascórbico (AA)}

Os teores de ácido ascórbico, ou vitamina $\mathrm{C}$, estão expressos na Figura 6. Para todas as potências, o teor de ácido ascórbico praticamente manteve-se constante e próximo ao do resíduo in natura durante os 10 primeiros minutos de desidratação, quando as taxas de umidade ainda são consideradas elevadas. Após isso, notou-se uma tendência de aumento desse composto, conforme o tempo de desidratação foi aumentando e o material atingia valores menores de umidade, obtendo-se valores muito superiores aos do resíduo antes da desidratação. Mais uma vez a potência de $480 \mathrm{~W}$ se mostrou a mais eficiente, atingindo os melhores resultados dentre todos os experimentos.

Dessa forma, verificou-se que apesar do exposto por Bobbio; Bobbio (1995), que afirmaram que o ácido ascórbico apresenta certa sensibilidade à temperatura e luz, obteve-se um efeito benéfico da exposição às micro-ondas sobre os teores desse composto, elevando os mesmos para os valores superiores aos do material in natura.

Isto pode ser explicado pelo fato de que além do rompimento das fibras que expõem o ácido ascórbico presente na matriz do material, o aumento da temperatura pode ter contribuído para a desativação de enzimas responsáveis pela degradação do mesmo, elevando seus teores, como observado por Dorta et al. (2012).

Figura 6 - AA para as desidratações por microondas: $280 \mathrm{~W}$ (a), $480 \mathrm{~W}$ (b), $600 \mathrm{~W}$ (c) e $800 \mathrm{~W}(\mathrm{~d})$.

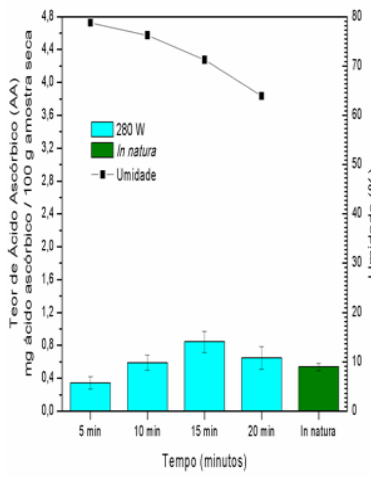

(a)

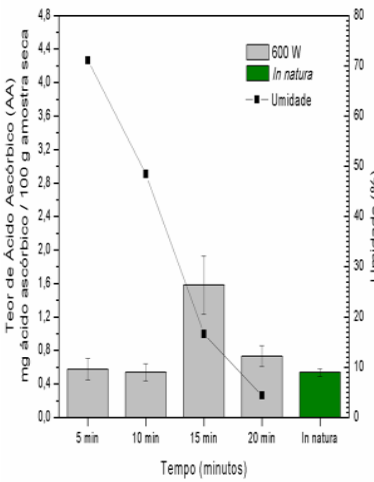

(c)

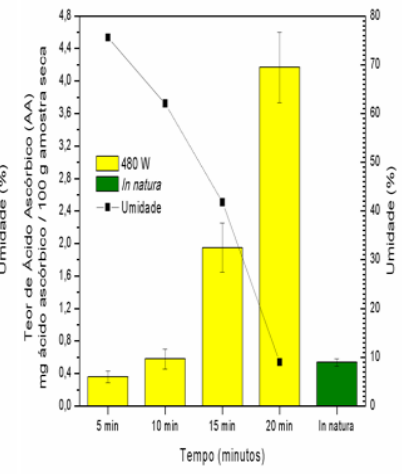

(b)

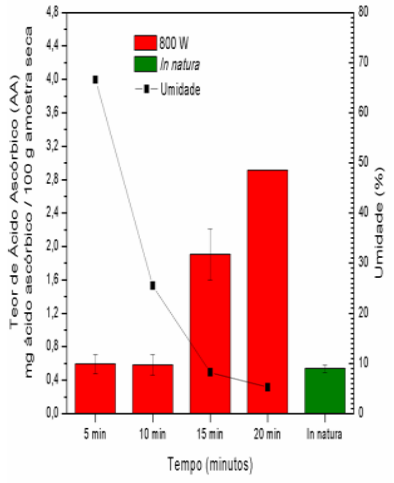

(d)

\subsection{Microscopia Eletrônica de Varredura (MEV)}

As imagens obtidas pela microscopia eletrônica de varredura (MEV) para o resíduo desidratado por micro-ondas estão expressas na Figura 7. Verificou-se através das mesmas que o aumento de potência impactou diretamente a estrutura interna do material, com era esperado. Foi possível observar que, conforme $\mathrm{o}$ resíduo foi submetido a intensidades de exposição cada vez maiores, sua estrutura foi tornando-se mais porosa, o que permitiu a liberação dos compostos 
bioativos, mas tal aumento foi limitado, ocorrendo um enrugamento e encolhimento da mesma após a exposição excessiva a radiação de micro-ondas.

Figura 7 - MEV para desidratações em $280 \mathrm{~W}$ (a), $480 \mathrm{~W}$ (b), $600 \mathrm{~W}$ (c) e $800 \mathrm{~W}$ (d) após 20 minutos e ampliação de 500 vezes.

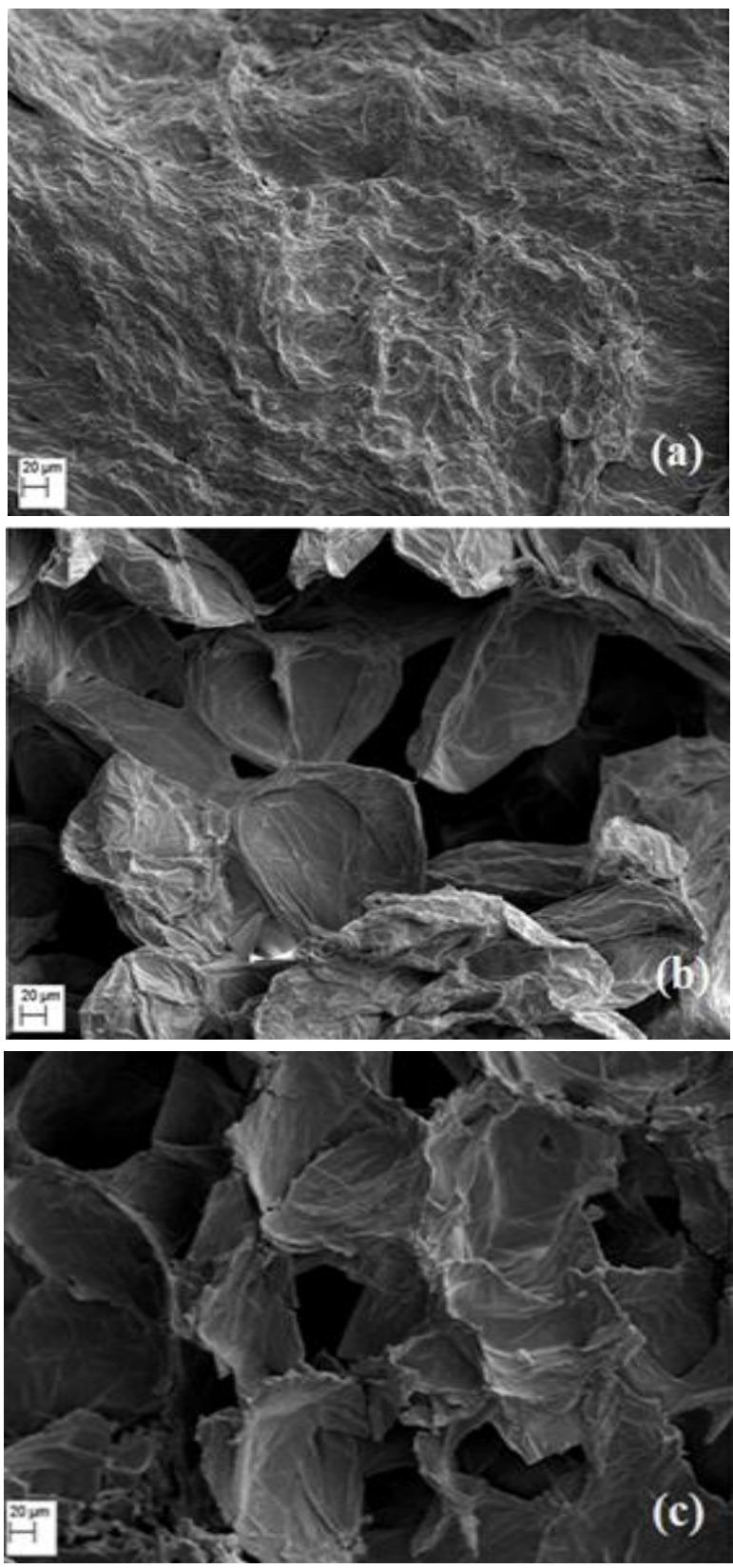

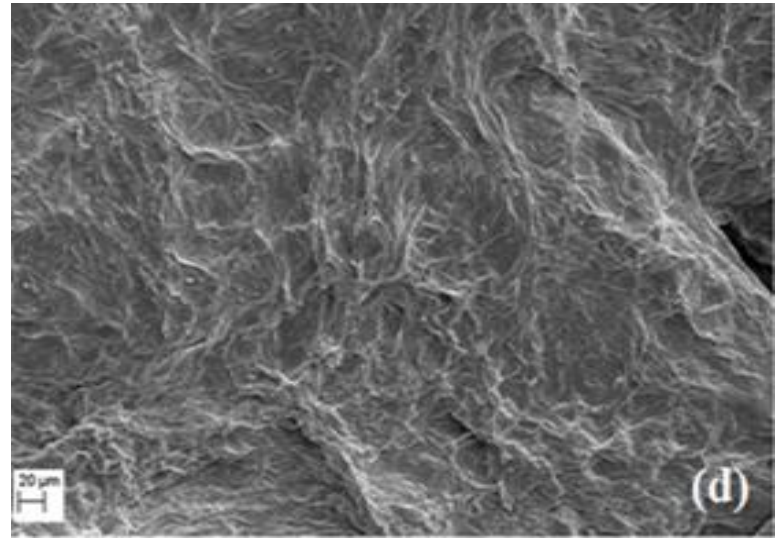

Dessa forma obteve-se uma estrutura levemente impactada pela desidratação na potência de $280 \mathrm{~W}$ (a), um aumento de poros considerável na potência de 480 W (b), considerada a mais adequada para a operação, o início da redução da porosidade na potência de $600 \mathrm{~W}$ (c) e por fim o encolhimento e enrugamento da estrutura devido às temperaturas excessivas obtidas na potência de $800 \mathrm{~W}$ (d).

\section{CONCLUSÕES}

Através dos experimentos realizados, foi possível concluir que a desidratação por micro-ondas se mostrou bastante eficiente na remoção de umidade do material, permitindo, em tempos reduzidos, obter-se produtos com baixos teores de água residual.

Apesar disso, verificou-se que para se obter um produto final de qualidade, é necessário que o processo seja conduzido em condições intermediárias de potência (cerca de $480 \mathrm{~W}$ ), permitindo maximizar o efeito positivo da desidratação, com valores elevados para os compostos fenólicos, flavonoides e ácido ascórbico. Os teores de ácido cítrico, independente da potência empregada, foram reduzidos após a desidratação.

Dessa forma, pode-se afirmar que o uso de micro-ondas na secagem de resíduos de maracujá é uma alternativa bastante viável 
para um possível aproveitamento futuro desse material, se destacando como um método muito eficiente desde que controlado adequadamente.

\section{REFERÊNCIAS}

AJILA, C. M.; BHAT, S. G.; PRASADA RAO, U. J. S. Valuable components of raw and ripe peels from two Indian mango varieties. Food Chemistry, v. 102, p. 10061011, 2007.

AOAC. Official Methods of Analysis. Association of Official Analytical Chemists, Gaithersburg, MD, 1995.

ARBALLO, J. R.; CAMPANONE, L. A.; MASCHERONI, R. H. Modeling of Microwave Drying Fruits. Drying Technology, v. 28, p. 1178-1184, 2010.

BOBBIO, F. O; BOBBIO, P. A. Introdução a química de alimentos, $2^{\mathrm{a}} \mathrm{Ed}$. São Paulo, 1995.

BORTOLOTTI, C. T., Estudo Experimental da Fluidodinâmica de Mistura de Resíduo de Acerola e Soja em Leito de Jorro. Dissertação de Mestrado, Programa de PósGraduação em Engenharia Química, Universidade Federal de Uberlândia (UFU), Uberlândia-MG, 2012.

CHANG, C. H.; LIN, H. Y.; CHANG, C. Y.; LIU, Y. C. Comparisons on the Antioxidant Properties of Fresh, Freeze-Dried and HotAir-Dried Tomatoes. Journal of Food Engineering, v. 77, p. 478-485, 2006.

DATTA, A. K.; ANANTHESWARAN, R.C. Handbook of Microwave Technology for Food Applications; Marcel Dekker: New York, 2001.
DORTA, E.; LOBO, M. G.; GONZALEZ, M. Using drying treatments to stabilize mango peel and seed: effect on antioxidant activity. LWT, v. 45, p. 261-268, 2012.

GHANEN, N.; MIHOUBI, D.; KECHAOU, N.; MIHOUBI, N. B. Microwave dehydration of three citrus peel cultivars: effect on water and oil capacities, color, shrinkage and total phenols content. Industrial Crops and Products, v. 40, p. 167-177, 2012

GUPTA, M.; WONG, W. L. E. Microwaves and metals. Singapore: John Wiley \& Sons (Asia) Pte. Ltd., 2007.

IBGE - Instituto Brasileiro de Geografia e Estatística. Produção Agrícola Municipal 2012. Disponível em: https:// www.embrapa.br/mandioca-e-fruticultura/ cultivos/maracuja, acessado em 25 de março de 2015.

IBRAF - Instituto Brasileiro de Frutas. Panorama da Cadeia Produtiva das Frutas 2012 e Projeções para 2013. Setembro/2013.

LI, Z. ; RAGHAVAN G. S. V.; ORSAT, V.; Optimal power control strategies in microwave drying. Journal of Food Engineering, v. 99, p. 263-268, 2010.

MANACH, C.; SCALBERT, A.; MORAND, C.; RÉMÉSY, C.; JIMÉNEZ, L. Polyphenols: food sources and bioavailability. American Journal of Clinical Nutrition, v. 79, n. 5, p. 727-747, 2004.

MASKAN, M. Microwave/air and microwave finish drying of banana. Journal of Food Engineering, v. 44, p. 71-78, 2000.

METAXAS, A. C., MEREDITH, R. J. Industrial microwave heating. London: Peter Peregrinus Ltd, 1983. 
MOUSA, N.; FARID, M. Microwave vacuum drying of banana slices. Drying Technology, v. 20, p. 2055-2066, 2002.

OLIVEIRA, L. F.; NASCIMENTO, M. R. F.; BORGES, S. V.; RIBEIRO, P. C. N.; RUBACK, V. B. Aproveitamento alternativo da casca do maracujá-amarelo (Passiflora edulis f. var. flavicarpa) para produção de doce em calda. Ciência e Tecnologia de Alimentos, Campinas-SP, v. 22(3), p. 259262, 2002.

PEREIRA, F. A.; CARNEIRO, M.R.; ANDRADE, L.M. A cultura do maracujá. Brasília, DF: Embrapa Informações Tecnológicas, 124 p. 2006.

PODSEDEK, A. Natural Antioxidants and Antioxidant Capacity of Brassica Vegetables: A review. LWT: Journal of Food Composition and Analysis, v. 40, p.1-11, 2007.

REY, L. Fundamental aspects of lyophilization. Aspects Théoriques et Industriels de la Lyophilization. Paris, Hermann, p. 23-43, 1964.

SILVA, P. B. Secagem de Resíduos de Frutas em Secador Roto-Aerado. Dissertação de Mestrado. Programa de PósGraduação em Engenharia Química, Universidade Federal de Uberlândia, Uberlândia-MG, 2014.

SINGLETON, V. L.; ROSSI, J. A. Colorimetry of total phenolics with phosphomolibidic_phosphotungistic acid reagents. American Journal of Enology and Viticulture, v. 16, p. 144-158, 1965.

VADIVAMBAL, R.; JAYAS, D. S. Nonuniform temperature distribution during microwave heating of food materials - A review. Food and Bioprocess Technology, v.3, p. 161-171, 2010.

VENKATESH, M. S.; RAGHAVAN, G. S. V. An Overview of Microwave Processing and Dielectric Properties of Agri-food Materials, Biosystems Engineering, v. 88(1), p. 1-18, 2004.

WOLFE, K.; WU, X.; LIU, R. H. Antioxidant activity of apple peels. Journal of Agricultural and Food Chemistry, v. 53, p. 609-614, 2003.

ZAREIN, M.; SAMADI, S. H; GHOBADIAN, B. Investigation of microwave dryer effect on energy efficiency during drying of apple slices. Journal of the Saudi Society of Agricultural Sciences, v. 14, p. 41-47, 2015.

ZHISHEN, J.; MENGCHENG, T.; JIANMING, W. The Determination of Flavonoid Contents in Mulberry and their Scavenging Effects on Superoxide Radicals. Food Chemistry, v.64, p. 555-559, 1999. 